\section{Asociación entre maloclusiones y trastornos del lenguaje}

\section{Association between malocclusions and language disorders}

\section{Resumen}

Entre las enfermedades bucodentales de mayor prevalencia se encuentran las maloclusiones, y dentro de los trastornos más comunes del desarrollo del lenguaje está la dislalia que corresponde a problemas de articulación en los sonidos sin base neurológica. Se realizó una revisión bibliográfica sobre el tema con el objetivo de describir la asociación entre problemas de fonación y maloclusión dental, específicamente identificar las alteraciones en la fonación más comunes, en pacientes con problemas de maloclusión dental. Dentro de los artículos revisados se excluyeron aquellos que asociaban trastornos del lenguaje a algún síndrome. Se puede concluir que la mayoría de los autores muestran una relación entre maloclusiones y trastornos de la articulación del habla, siendo la mordida abierta la anomalía más prevalente.

Palabras clave: Trastornos de la articulación; Lenguaje; Mordida abierta; Mordida cruzada (fuente: DeCS BIREME).

\begin{abstract}
Malocclusions are listed among the most prevalent oral diseases; whereas dyslalia is considered the most common speech disorder that involves articulation problems while producing sounds, without neurological basis. A literature review was performed on the topic, in order to describe the correlation between phonation problems and dental malocclusions, specifically identifying the most common phonation issues in patients with dental malocclusion problems. Within the articles reviewed, those where language disorders were associated to any syndrome, were excluded. The corollary after the review revealed that most of the authors demonstrated a relation between malocclusions and speech disorders, with open bite being the most prevalent anomaly.
\end{abstract}

Keywords: Articulation disorders; Language; Open bite; Cross bite (source: MeSH NLM).

\section{Artículo de Revisión}

Lucía Lorena Bravo Rivera ${ }^{1, a}$, Katherine Johana Gerbert Castilo $^{2, b}$, Giovanna Margarita Salas Toloza ${ }^{1, b}$

${ }^{1}$ Universidad de Concepción, Facultad de Odontología. Concepción, Chile.

2 Universidad Mayor, Facultad de Odontología. Temuco, Chile.

a Magíster en Educación Médica.

${ }^{b}$ Cirujano Dentista.

\section{Correspondencia:}

Lucía Lorena Bravo Rivera

Correo electrónico: luciabravo@udec.cl

Avenida Roosevelt 1550. Concepción, Chile.

Código postal: 4070369

\section{Coautores:}

Katherine Johana Gerbert Castilo

Katherine.gerbert@outlook.com

Giovanna Margarita Salas Toloza

gisalastoloza@gmail.com

Editor:

Yuri Castro-Rodríguez

Universidad Nacional Mayor de San Marcos, Perú.

Conflicto de intereses: los autores declaran no tener conflictos de interés.

Fuente de financiamiento: autofinanciado.

Recibido: 16/01/19

Aceptado: 20/02/19

Publicado: 05/06/19 


\section{Introducción}

La oclusión dental forma parte del sistema masticatorio o estomatognático en la que se incluyen dientes, tejidos de soporte, sistema neuromuscular, articulaciones temporomandibulares y esqueleto craneofacial. Por consecuencia, el concepto de oclusión ideal se entiende como la relación funcional existente entre los componentes del sistema masticatorio ${ }^{1}$.

Dentro de las enfermedades bucodentales más prevalentes a nivel mundial se encuentran las maloclusiones ${ }^{2}$, esta es el resultado de la anormalidad morfológica y funcional de los componentes óseos, musculares y dentarios que conforman el sistema estomatognático. Los factores genéticos y el medioambiente son los dos factores principales ${ }^{3}$.

El Diagnóstico Nacional de Salud Bucal indicó que estas alteraciones en Chile presentan una prevalencia de $52,6 \%$ a los 12 años de edad ${ }^{4}$. Es por ello que, debido a la alta prevalencia de esta alteración en la población, se debe conocer las complicaciones funcionales que puede traer consigo, entre las que se incluyen las alteraciones del habla ${ }^{5}$.

El habla es un proceso que puede ser modificado por diversos factores, ya que los sonidos que se transforman en palabras se generan debido a la articulación de labios, dientes, paladar y lengua ${ }^{6}$, por esto es importante la armonía del ambiente dental, que influye en la postura y acomodo de la lengua al hablar y consecuentemente en la calidad de la articulación de los fonemas ${ }^{7}$. Al existir un desequilibrio dental e instaurarse una maloclusión es posible que se afecte la función, lo que conllevará a fallas del habla.

Entre los trastornos evidentes de la fonación donde interviene la dinámica de los órganos fonoarticuladores se encuentran las dislalias ${ }^{8}$. Etimológicamente la palabra dislalia significa dificultad en el habla (del griego dis, dificultad y lalein hablar). La dislalia abarca los trastornos de la articulación en los sonidos del habla (pronunciación) en los que no exista como base una entidad neurológica ${ }^{9}$, se presentan con mayor frecuencia entre los 5 y 7 años, y muchas veces es pasajera.

La presente revisión plantea como propósito conocer la asociación existente entre los problemas de dislalias y de malocusión dental y como objetivo específico identificar las alteraciones en la fonación más comunes en pacientes con problemas de maloclusión dental.

\section{Desarrollo}

En la búsqueda de exponer la asociación entre problemas de fonación y maloclusión dental, se realizó una revisión de 31 referencias bibliográficas. Los criterios de inclusión fueron seleccionar revisiones y casos clínicos que tratasen estos términos. Se excluyeron aquellos artículos en los que se haya abordado trastornos del lenguaje como parte de algún síndrome o acompañados con algún trastorno cognitivo basal.
Dentro de la vasta información considerada, cabe destacar que la cavidad bucal es una estructura en la que en forma conjunta se llevan a cabo múltiples funciones, como son: la primera fase de la digestión, deglución, masticación, articulación y fonación. En la que la fonación es el acto de emitir el sonido de la voz (voluntario), la fonoarticulación es hacer audible el lenguaje, para que este sea lenguaje oral y finalmente el habla es la parte articulada del lenguaje oral ${ }^{10}$.

El "habla", es un proceso fisiológico que permite hacer uso del lenguaje y materializarlo mediante comunicación verbal, se lleva a cabo por medio del aparato fonoarticulatorio que genera un mecanismo complejo y dinámico donde intervienen órganos móviles: labios, lengua y velo del paladar, a los que se le atribuyen las particularidades más importantes de una correcta articulación, y órganos fijos, constituidos fundamentalmente por los dientes ${ }^{11}$.

Existen básicamente cuatro tipos de alteraciones del habla: la disartria (trastorno de la articulación del habla asociado a un daño neurológico), la disfemia (trastorno del ritmo y fluidez del habla), la disglosia (trastorno de la articulación del habla, debido a una alteración anatómica y funcional de los órganos periféricos activos y pasivos que intervienen en el habla) y la dislalia; siendo entre estas, la dislalia la alteración más frecuente entre los escolares ${ }^{12}$. Las dislalias se definen como un trastorno de la articulación de los fonemas, el cual es el resultado de la incapacidad para pronunciar o formar correctamente ciertos fonemas o grupo de estos, en los que no existe una base etiopatogénica de índole neurológico.

Las dislalias se clasifican etiológicamente en cuatro tipos:

Evolutivas. Alteraciones articulatorias que se manifiestan en edades de 3 a 4 años y son propias del desarrollo evolutivo de los niños. Tienen un carácter fisiológico y forman parte del aprendizaje normal del lenguaje.

Audiógenas. Alteraciones que se deben a la falta de una buena audición. Si la falta de audición es parcial como la hipoacusia, antes o después de establecido el lenguaje, pueden aparecer los trastornos en la articulación. Una hipoacusia dificulta el reconocimiento y la reproducción de sonidos con similitud fonética.

Orgánicas. Trastornos de la articulación originados por malformaciones anatómicas. También son conocidas como disglosias, dentro de las cuales podemos distinguir:

- Disglosia labiales: en este caso los problemas a la hora de realizar una correcta pronunciación son consecuencia de una alteración en la forma, movilidad o fuerza de los labios.

- Disglosia mandibular: en este caso las dificultades a la hora de articular vienen dadas por una alteración en la forma de los maxilares -ya sea solo en uno o en ambos-.

- Disglosia lingual: dificultad a la hora de realizar una correcta pronunciación debido a una alteración orgáni- 
ca de la lengua que influye negativamente en la rapidez y precisión de los movimientos de la lengua.

- Disglosia palatina: conlleva problemas a la hora de articular los fonemas como consecuencia de una alteración orgánica del paladar óseo o del velo del paladar.

- Disglosia dental: origina problemas a la hora de pronunciar debido a una mala posición, forma o presencia de dientes.

Funcionales. Alteraciones en la articulación de la palabra, debidas a una mala coordinación de los movimientos que son necesarios para articular ciertos fonemas. No existe ningún trastorno físico u orgánico que las justifiqué, sino que solo una incapacidad funcional, fundamentalmente inducida por: educación deficiente, circunstancias sociales desfavorables e incoordinación motora.

Las dislalias se pueden presentar como tres tipos de alteraciones:

- Sustitución: error en la articulación en la cual un sonido es reemplazado por otro, aquí el niño emite un sonido parecido al que corresponde, pero incorrecto. Puede darse al principio, en medio o al final de la palabra, por ejemplo, "cardo", en lugar de carro".

- Omisión: dificultad que presenta el niño para pronunciar un fonema, donde suele omitir los fonemas que le resultan difíciles, como por ejemplo, dice "lápi", en lugar de decir "lápiz".

- Distorsión: error en la articulación en la cual se da de forma incorrecta o deformada, lo que hace es sustituir un sonido por otro. Generalmente se presenta debido a una incorrecta posición de los órganos de la articulación, a la forma de salida de aire, o a la vibración (o falta de esta) de las cuerdas vocales, por ejemplo, dice "tasa", en lugar de decir "casa" ${ }^{13}$.

Con base a lo anterior, se puede señalar que la presencia de alguna anomalía dentomaxilar en niños, conseguiría condicionar la instauración de trastornos del habla ${ }^{14} \mathrm{y}$ si bien se han publicado estudios para demostrar una relación entre la existencia de maloclusiones y trastornos del lenguaje, se puede encontrar evidencia que manifiesta lo contrario; Peña et al. ${ }^{9}$ en una investigación realizada en Colombia, en la que se estudió la prevalencia de maloclusiones en pacientes dislálicos, encontró que el 68,3\% de estos presentaban algún tipo de maloclusión, es decir un $32 \%$ restante presentaba un trastorno articulatorio, sin presencia de maloclusión. De esta manera se puede corroborar que los problemas del habla no siempre van acompañados de maloclusión dental. Por otro lado, este mismo estudio logró establecer una relación entre, mordida abierta anterior Clase III de Angle con overjet disminuido y problemas de apiñamiento con dislalias, sin embargo, esta relación no fue estadísticamente significativa. Resultados similares se reportan en un estudio realizado en México por Taboada et al. ${ }^{11}$, cuyo objetivo al igual que el anterior, era determinar las prevalen- cias de trastornos del habla y maloclusión, donde no se logra establecer una relación concluyente entre ambos trastornos, en el cual, de un total de 93 niños revisados con un promedio de edad de 5 años, un $61 \%$ presentó maloclusiones y un $42 \%$ trastornos del habla, si bien ambos problemas estuvieron presentes en un $40 \%$ de los niños, nuevamente no se logra establecer una relación significativa.

López et al. ${ }^{15}$, estudiaron en México la relación entre la pronunciación de fonemas con el grado de maloclusión y la posición de bordes incisales-borde bermellón del labio, no encontraron asociación entre el grado de maloclusión y pronunciación de fonemas, por lo que los autores indicaron que el problema no es una relación simple de causa y efecto, puesto que algunas de las maloclusiones no provocan anomalías fonéticas discernibles debido al mecanismo de ajuste del sistema estomatognático, el que no se puede dejar de considerar al realizar este tipo de estudios. Por otra parte, en busca de una relación entre dimensiones del arco maxilar y trastornos del habla, Heliövaara ${ }^{16}$ realizó un estudio en 52 niños de 6 años en Finlandia, con problemas en la articulación de los fonemas $/ \mathrm{r} /, / \mathrm{s} /$ y $/ \mathrm{l} /$; en su estudio la relación no fue estadísticamente significativa con una longitud del arco maxilar más pequeña que el grupo de control.

Aun cuando existe evidencia que avale la poca o ninguna asociación entre la existencia de maloclusiones y trastornos del lenguaje, un estudio realizado por Wauters et al. ${ }^{17}$ en la comuna de Cochamó en Chile, plantea lo contrario. Ellos determinaron las prevalencias de anomalías dentomaxilares y de trastornos articulatorios, donde se puede ver que la prevalencia de maloclusión alcanzó el $89,7 \%$ (sobre la frecuencia de los estudios realizados en Chile), y para trastornos articulatorios de 30\%. Dentro de los fonemas que presentaron mayor dificultad fueron $/ \mathrm{r} /, / \mathrm{rr} / \mathrm{y} / \mathrm{s} /$, asociado a niños con resalte aumentado o mordida abierta, esto se explica, de acuerdo a Wauters et al. ${ }^{17}$ a que la biofisiodinamia articulatoria de estos fonemas, requieren una buena relación entre las arcadas dentarias, así como un adecuado apoyo lingual. En relación a esta asociación, Ocampo-Parra et al. ${ }^{18}$ muestran de igual forma que el estudio anterior, que la mordida abierta está fuertemente asociada a desórdenes en la pronunciación de fonemas y de acuerdo a sus resultados, un $75,8 \%$ de pacientes que presentan mordida abierta anterior presentan dislalia, aquí la mayor dificultad de sonidos fue en aquellos fonemas en los cuales para ser emitidos necesitan de articulación dental. Es así que los fonemas / $\mathrm{t} / \mathrm{y} / \mathrm{d} /$ tienen lugar en la articulación dental; /s/ es alveolar; $\mathrm{y} / \mathrm{ch} / \mathrm{y} / \tilde{n} / \mathrm{son}$ palatales. Además, uno de los hallazgos observados en este estudio fue que la distorsión fonética de estos fonemas modifica la posición de la lengua, causando empuje interdental de la lengua, que puede conducir a la perpetuación y recurrencia de mordida abierta anterior aún después de lograda la corrección con tratamiento ortodóncico.

En otro estudio realizado en Chile, por Herrero ${ }^{19}$ en la comuna de Juan Fernández se encontró que los niños con anomalías dentomaxilares presentaron con mayor 
frecuencia alteraciones en la articulación de fonemas. El porcentaje de niños con alteraciones fonoarticulatorias fue de $57,9 \%$, todos ellos presentaron interposición lingual en la articulación de al menos un fonema postdental. Los niños con anomalías, especialmente con mordida abierta o compresión con mordida cruzada, presentaron con mayor frecuencia alteraciones en la articulación de estos fonemas. Esto se debería a que al perderse la oclusión normal o el correcto alineamiento dentario, los puntos articulatorios de referencia habitual sufrirían modificaciones.

En este mismo sentido, Leavy et al. ${ }^{20}$ en el 2015 realizaron un estudio en 115 sujetos de entre 8 y 36 años de edad, de origen hispano radicados en el estado de Nueva York, Estados Unidos, que hablaban en inglés, donde el $32 \%$ hablaba español como idioma principal en el hogar, y encontraron que existe una fuerte asociación entre los diferentes tipos de maloclusiones y alteraciones en el habla; que mientras más severa es la maloclusión mayor es la probabilidad que ocurran errores en la producción de fonemas. Pacientes con mordida abierta anterior (mayor a $2 \mathrm{~mm}$ ) tienen mayores problemas de pronunciación sobre todo en los fonemas $/ \mathrm{s} / \mathrm{y} / \mathrm{t} /$; además, esto se ve agravado en pacientes que también presenten mordida cruzada posterior.

Laine et al. ${ }^{21}$ por su parte, sostienen que un diastema anterior de los incisivos maxilares se asocia a problemas en pronunciación de sonidos de "l", "n", "d" y "r"; $\sin$ embargo, afirman también que el aumento de mordida abierta y overjet juegan un rol más importante si lo comparamos con diastema. Así mismo, Orozco et al. ${ }^{22}$ en un estudio realizado en México a 156 pacientes dice que las anomalías de la oclusión dentaria son la causa más frecuente de dislalias orgánicas. El resalte aumentado y los diastemas, fueron las maloclusiones que con mayor frecuencia provocaron alteraciones articulatorias; las dislalias funcionales fueron las que se presentaron con mayor frecuencia. Se encontró, además, una gran relación entre las anomalías de la oclusión y la presencia de hábitos deformantes bucales que pueden ser prevenidos en la atención primaria.

Para valorar la percepción que los padres y/o tutores tienen de los trastornos en el habla de sus niños, un estudio realizado en Brasil por Pereira et al. ${ }^{23}$ evaluó, a través de la aplicación de un cuestionario a padres, la percepción de alteraciones en el sistema estomatognático de sus hijos, donde no se hizo distinción en los tipos de trastornos del habla, se observó que los pacientes que presentaban mordida abierta anterior y además el mal hábito de onicofagia, luego de los 2 años de vida, presentan alteraciones fonéticas producidas principalmente por la anteriorización de la lengua entre los arcos dentales, cambiando la producción de los fonemas linguo-dental /t/, /d/, /n/ y /l/ y fonemas linguo-alveolar /s/y /z/. Esto concuerda con el estudio realizado por Ocampo-Parra et al. ${ }^{18}$ el cual indica que pacientes que presentan mordida abierta anterior tienen mayor prevalencia de dislalias; Farronato et al. ${ }^{24}$ indica que existe una estrecha relación entre maloclusiones y dislalias, además, que estas a menudo influyen en las maloclusiones y estas últimas siempre agravan las malas articulaciones fonéticas. Rivadeneira et al. ${ }^{25}$, determinó una relación evidente entre los trastornos del habla y la maloclusión dental, además se mostró que el 36,1\% los niños de 5 años de edad con alguna maloclusión dental presentaban dislalias y, que a los de 6 años el 25\% las presentaban. Sin embargo, cabe destacar que estas combinaciones no se dan en todos los casos, por lo cual se podría deducir que también hay otros factores asociados que pueden dar origen a cualquiera de las dos enfermedades. Además, destacaron que mientras más grave sea la maloclusión, mayor es la severidad del trastorno del habla. Por lo cual, si se está en presencia de clase III, diastema, aumento de overjet, presencia de mordida abierta y mordida profunda existe una alta tendencia a estar asociado con trastornos del habla, entre ellas las dislalias. La presencia de apińamiento y mordidas cruzadas anteriores tienen una tendencia moderada a asociarse con tales trastornos; y por el contrario oclusión clase II, protrusión de incisivos superiores, mordida cruzada posterior y alteraciones temporomandibulares, tienen baja tendencia a asociarse con dislalias.

A pesar de lo anterior, se sabe que hay pacientes que presentan maloclusiones graves y que no presentan alteraciones del lenguaje, lo que puede ser explicado a través de la compensación fisiológica de la deformidad anatómica que hace que la función sea posible.

En busca de la prevalencia de defectos del habla en pacientes diagnosticados con maloclusiones dentales en los tres planos del espacio, Benavides et al. ${ }^{26}$, obtienen en su estudio, que la mayor prevalencia fue la linguoalveolar $(98,5 \%)$, donde la totalidad de los pacientes diagnosticados con defectos del habla presentaron algún tipo de maloclusión en el plano sagital; en el plano vertical fue la mordida abierta anterior la más predominante, y en el plano transversal la mordida cruzada $(19,1 \%)$. Los defectos del habla de las sílabas /cr/, /gr/ y /pr/ se relacionaron con la maloclusión clase III, lo que sugiere que las maloclusiones son un factor de riesgo para la presencia de dislalias.

Resultados semejantes son los reportados en el estudio de Villanueva et al. ${ }^{27}$, donde se encontró mayor porcentaje de modificación en los fonemas medios $/ \mathrm{d} /, / \mathrm{s} /$ y /t/ para todas las clases con la siguiente proporción: Clase I, 14,9\%; Clase II, 27.6\% y Clase III, 20,2\%. Las asociaciones entre los trastornos del habla y la capacidad de movimiento mandibular, así como también con la presencia de maloclusiones, según la investigación presentada por Pahkala et al. ${ }^{28}$, indican que es probable que las personas con trastornos del habla tengan una capacidad de apertura menor, y que esta disminuye en la edad adulta, y que la capacidad de movilidad mandibular se vería afectada especialmente en casos de sobremordida excesiva, mordida cruzada lateral y mordida abierta anterior, por lo que se favorecía la presencia de dislalias.

Entre los autores que relacionaron la presencia de hábitos orales con las dislalias, se encuentran Cab et al. ${ }^{29}$ quienes reportan que los hábitos más frecuentes 
son: succión digital, respiración bucal, rinolalia, deglución atípica, succión de labios, succión de chupetes y biberón.

Para la relación entre la pérdida prematura de piezas dentales anteriores y la articulación de fonemas fricativos /f/, /j/, /s/, /z/ e /y/, Benavente y Ghezzi ${ }^{30}$, encontraron que existe una asociación estadísticamente significativa entre los fonemas /f/, /s/ y /z/, pero no para los fonemas /j/ e $/ \mathrm{y} /$. Antecedentes similares fueron presentados por Moyers ${ }^{31}$, quien refiere que las consonantes fricativas son las más afectadas porque requieren una ubicación muy precisa de los órganos de la dicción, y por lo tanto en su pronunciación resultarían ser las más defectuosas. Este estudio comprueba lo antes mencionado, sin embargo, determina que no todos los fonemas son afectados por la pérdida prematura de las piezas antero-superiores, solo los fonemas $/ \mathrm{f} /, \mathrm{s} / \mathrm{y} / \mathrm{z} /$.

Por último, para determinar la relación entre dislalias y maloclusión dental, Vásquez-Reyes et al. ${ }^{12}$ con apoyo de la prueba TEPROSIF aplicada en una muestra de 116 escolares de 4 a 6 años de edad, encontraron resultados que permiten establecer una correlación significativa entre "plano terminal y omisión y sustitución", entre "arco de Baume tipo I y omisión", "tipo de mordida y sustitución" y "ausencia de dientes y omisión y sustitución", observando que no hubo una diferencia significativa en relación al sexo de los niños evaluados. La sustitución de fonemas es la alteración más frecuente; esta va seguida de la omisión de fonemas y, por último, la distorsión de fonemas. Así mismo Álvarez et al. ${ }^{10}$, en una evaluación a 50 nińos menores de 5 años, pudieron observar que existe asociación significativa entre los planos terminales mesial exagerado, distal, y mordida abierta y los trastornos del habla, siendo el mayor trastorno del habla la sustitución. El mayor trastorno del habla en sobremordida vertical y horizontal es la omisión. Además, en relación a los fonemas, los que se presentaron con 'mayor distorsión fueron $/ \mathrm{r} /, / \mathrm{rr} / \mathrm{y} / \mathrm{s} /$ ', 'mayor omisión fue $/ \mathrm{d} /, / \mathrm{l} / \mathrm{y}$ $/ \mathrm{r} /$ ' y 'mayor sustitución fueron $/ \mathrm{dxl} /$, / $/ \mathrm{xr} /$, , /xrr/'.

\section{Conclusiones}

Los problemas del habla se encuentran tanto en personas con maloclusión dental como en los que tienen una oclusión normal. Por otro lado, puede haber una persona con maloclusión sin desarrollo de trastorno del habla.

De acuerdo a la revisión bibliográfica, se puede concluir que la mayoría de la literatura consultada postula que existe una relación entre maloclusión y trastornos del habla, siendo la anomalía más asociada a esto la mordida abierta.

Finalmente, se puede observar que existen pocos estudios realizados recientemente con respecto a los trastornos articulatorios y su relación con maloclusiones, por lo que resulta necesario que se desarrollen más investigaciones sobre el tema en Sudamérica; ya que de esta manera podemos fomentar un trabajo interdisciplinario dentro de los equipos de salud, sobre todo la relación entre fonoaudiólogo y odontólogo.

\section{Referencias bibliográficas}

1. Firmani M, Becerra N, Sotomayor C, Flores G, Salinas JC. Oclusión terapéutica. Desde las escuelas de oclusión a la Odontología Basada en Evidencia. Rev. Clin. Periodoncia Implantol. Rehabil. Oral. 2013;6(2):90-95.

2. Sheiham A. Oral health, general health and quality of life. Bulletin of the World Health Organization. 2005;83(9):644.

3. García García VJ, Ustrell Torrent JM, Sentís Vilalta J. Evaluación de la maloclusión, alteraciones funcionales y hábitos orales en una población escolar: Tarragona y Barcelona. Av Odontoestomatol. 2011;27(2):75-84.

4. Ministerio de Salud Chile. Diagnóstico nacional de salud bucal del adolescente de 12 años y evaluación del grado de cumplimiento de los objetivos sanitarios de salud bucal 2000-2010. Informe de facultad de Odontología Universidad Mayor. Chile: FONIS MINSAL-CONICYT; 2007. Serie documentos técnicos año 2007.

5. Schonhaut L, Maggiolo M, de Barbieri Z, Rojas P, Salgado A. Dificultades de lenguaje en preescolares: Concordancia entre el test TEPSI y la evaluación. Rev Chil Pediatr. 2007;78: 369-375.DOI:10.4067/S037041062007000400004

6. Maggiolo M, Coloma C, Pavez M. Estimulación de narraciones infantiles. Rev. CEFAC. 2009; 11(3):379-388. DOI:10.1590/S1516-18462009005000043

7. Pizolato R, Fernandes F, Gavião M. Speech evaluation in children with temporomandibular disorders. J Appl Oral Sci. 2011;19(5):493-499.

8. Jiménez A, Acosta B, Soto C, Fernández L. Alteraciones del habla en niños con anomalías dentomaxilofaciales. Rev Cubana Ortod. 1997;13(1):29-36.

9. Peńa M, Rojas M, Tirado A, Benavides B, Hurtado M, Ruíz A. Prevalencia de la maloclusión en tres planos del espacio en pacientes diagnosticados con defectos del habla en las clínicas de la especialización de ortopedia funcional y ortodoncia de la Universidad Cooperativa de Colombia Prev Rev Estomatol. 2014;22(1):26-32.

10. Álvarez L, Oropeza P, Elorza H. Trastornos del habla asociados a maloclusión dental en pacientes pediátricos. Rev Odontol Mex. 2005;9(1):23-29.

11. Taboada-Aranza O, Torres A, Cazares C, Orozco L. Prevalencia de maloclusiones y trastornos del habla en una población preescolar del oriente de la Ciudad de México. Bol Med Hosp Infant Mex. 2011;68(6):425-430.

12. Vázquez-Reyes A, Reyes y Zepeda A, Moyaho-Bernal A, Moreno-García, A, Montiel-Jarquín, ÁJ, Hernández-Ruíz, AK, Bejarano-Huertas, R, López-Colombo, A. Dislalias asociadas a maloclusión dental en escolares. Rev Med Inst Mex Seguro Soc. 2014;52(5):538-542.

13. 27th World Congress of the International Association of Logopedics and Phoniatrics (IALP): Dinamarca, Clinical Analysis in Speech and Language Therapy: Occlusal Class and Speech Production. Copenhague; 5-9 agosto 2007. Lyngby: Danish Technological University, Lundtoft; 2007.

14. Calavia J. Estado de la cuestión: Los trastornos del habla en los primeros ańos de edad [tesis de licenciatura]. [Za- 
ragoza]: Facultad de Educación, Universidad internacional de la Rioja; 2014. 23p.

15. López S, Marichi F, Flores A, Ibarra D, Canseco J, Cuairán $\mathrm{V}$. Pronunciación de fonemas en relación con el grado de maloclusión y posición de bordes incisales-borde bermellón del labio. Rev Mex Ortod. 2016;4(4):219-226.

16. Heliövaara A. Maxillary Dental Arch Dimensions in 6-Year-Old Children with Articulatory Speech Disorders. Folia Phoniatr Logop. 2011;63:242-246. DOI:10.1159/000323436

17. Wauters M, Vergara D, Vergara A, Pérez D, Morollón J, Aguilera J. Prevalencia de anomalías dentomaxilares y articulatoria en escolares, Cochamó 2014. Rev Chil Ortod 2015;32(2):74-81.

18. Ocampo-Parra A, Escobar-Toro B, Sierra-Alzate V, Rueda Z, Lema M. Prevalence of dyslalias in 8 to 16 year-old students with anterior open bite in the municipality of Envigado, Colombia. BMC Oral Health. 2015;15:77. DOI:10.1186/s12903-015-0063-1

19. Herrero C. Anomalías dentomaxilares, malos hábitos orales y alteraciones fonoarticulatorias en la población endogámica del Archipiélago de Juan Fernández [tesis de licenciatura]. [Santiago]: Facultad de Odontología, Universidad de Chile; 2003. 76-81 p.

20. Leavy K, Cisneros G, Leblanc E. Malocclusion and its relationship to speech sound production: Redefining the effect of malocclusal traits on sound production. Am J Orthod Dentofacial Orthop. 2016;150(1):116- 123.

21. Laine T, Jaroma M, Linnasalo A-L. Articulatory disorders in speech as related to the position of the incisors. Eur J Orthod.1985;7:260-266.

22. Orozco L, Moreno W, Sánchez C, Álvarez A, Cardoso M, Moreno G. Articulación de las consonantes en malolcusión dental. Rev Esp Cienc Salud. 2012;15(1):26-29.
23. Pereira T, Oliveira F, Almeida M, Association between harmful oral habits and the structures and functions of the stomatognathic system: perception of parents/ guardians. CoDAS. 2017; 29(3). DOI:10.1590/2317$1782 / 20172015301$

24. Farronato G, Giannini L, Riva R., Galbiati G, Maspero C. Correlations between malocclusions and dyslalias. Eur J Paediatr Dent. 2012;13(1):13-18.

25. Rivadeneira J. Trastornos del habla asociados a maloclusión dental en estudiantes de primer y segundo grado de la Unidad Educativa Policía Nacional [tesis de licenciatura]. [Quito]: Facultad de Odontología, Universidad Central del Ecuador; 2017. 65 p.

26. Benavides B, Hurtado M, Ruíz A. Prevalencia de los defectos del habla en pacientes con maloclusiones dentales. Rev Investig Logop. 2017;2:189-202.

27. Villanueva P, Morán D, Loreto M, Palomino H. Articulación de fones en individuos clase esqueletal 1 , ll y 111 . CEFAC. 2009;11(3):423-430.

28. Pahkala RH, Qvarnström MJ. Mandibular movement capacity in 19-year-olds with and without articulatory speech disorders. Acta Odontol Scand. 2002;60:341345.

29. Cab A, Campechano E, Flores Y, López C, Zamora R, Reyes Z, et al. Dislalia asociada a hábitos orales. Oral. 2012;13(41):865-869.

30. Benavente J, Ghezzi L. Asociación entre el número de piezas dentarias del sector anterior-superior perdidas en forma prematura y los trastornos en la articulación de fonemas fricativos. Kiru. 2007;4(1):8-13.

31. Moyers, RE. Manual de Ortodoncia. Buenos Aires: Editorial Medica Panamericana; 1992. p. 19-42. 